\title{
EL COMERCIO ELECTRÓNICO COMO APOYO A LA COMERCIALIZACIÓN EN EMPRESAS DEL SECTOR REFRIGERACIÓN
}

\author{
E-commerce as support for marketing \\ in companies in the refrigeration sector
}

\author{
Diana Suárez López ${ }^{1}$, Rafael García Jiménez ${ }^{2}$ \\ Lucía Lizcano Flórez ${ }^{3}$
}

Recibido: Abril 8 de 2016/Aceptado: Julio 22 de 2016

\begin{abstract}
RESUMEN
El presente trabajo tiene como objetivo proponer estrategias que ayuden a fortalecer la comercialización de los productos y servicios pertenecientes al sector de la refrigeración en la ciudad de Barranquilla, a través de herramientas tecnológicas ofrecidas por Internet, haciendo uso del marketing interactivo y de esta manera apoyar la toma de decisiones hacia la gerencia. Como primera medida se hizo un análisis interno para diagnosticar la situación actual de las empresas objeto de estudio, con respecto al uso que se les da a las herramientas Web que emplean o si no hacen uso de estas y bajo que estrategias están manejando la mercadotecnia dentro de la organización.
\end{abstract}

Palabras clave: Marketing digital, Comercio electrónico, Herramientas Web.

\section{ABSTRACT}

The objective of this paper is to propose strategies that help strengthen the marketing of products and services belonging to the refrigeration sector in the city of Barranquilla, through technological tools offered by the Internet, using interactive marketing and thus supporting the decision making towards the management. As a first step an internal analysis was made to diagnose the current situation of the companies under study, with respect to the use given to the Web tools that they use or if they do not use them and under what strategies they are managing the marketing within of the organization.

Keywords: Digital marketing, Electronic commerce, Web Tools.

Cómo referenciar este artículo: Suárez, D., García, R. \& Lizcano, L. (2016). El comercio electrónico como apoyo a la comercialización en empresas del sector refrigeración. Ad-Gnosis, 5(5), 151-162.

1. Ingeniera de Sistemas. Especialista en Ingeniería de Software. Magíster en Administración de Empresas e Innovación. Docente investigadora, Universidad Simón Bolívar. orcid.org/0000-0002-5934-2545.

dsuarez@unisimonbolivar.edu.co

2. Magíster en Administración de Empresas e Innovación. Magíster en Estadística Aplicada, Universidad Simón Bolívar. rgarcia1@unisimonbolivar.edu.co

3. Licenciada en Mercadotecnia. Magíster en Administración Pública y Política Pública. Doctora en Ciencias Sociales. bucaliz@hotmail.com 


\section{Introducción}

Teniendo en cuenta que en muy pocos años el Internet se ha consolidado como una poderosa plataforma que ha cambiado el marketing $y$ el comercio, otorgándoles mayor dinamismo y una dimensión internacional; el Internet constituye el más tolerante e interactivo de todos los medios masivos de comunicación; con una muy baja inversión, le permite a cualquiera llegar en forma directa, rápida y económica al cliente, sin importar su ubicación geográfica; el desarrollo de las bases de datos. El Internet como medio de comunicación global y la utilización de la telefonía móvil como instrumento de comunicación, han propiciado un nuevo y espectacular desarrollo del marketing basado en la utilización de las nuevas TIC.

La aplicación de los negocios digitales hoy en día son el motor de avance y competitividad de las empresas del mundo. A través de ellos logran mejorar su fuerza de venta y reforzar las relaciones de sus clientes/proveedores, entre otras fortalezas. La competencia de las empresas en el mercado tiende hacia el uso de las Tecnologías de Información (TI). La Web nos permite abarcar unos de los aspectos más importantes en el éxito de un negocio: Las ventas o transacciones y manejo de clientes y/o proveedores. En ese sentido Berenguer y Ramos (2003) afirman que la mejora de competitividad de las empresas pasa por la innovación y la mejora de la gestión de sus procesos de negocios utilizando TI.

Los públicos y mercados en la Web están en continuo crecimiento, pero la presencia de las Pymes colombianas en la red de redes es aún incipiente. De acuerdo con el primer estudio Uso de Internet y Nuevas Tecnologías en Pymes colombianas exportadoras o potencialmente exportadoras, asegura que el $81 \%$ de las Pymes no están realizando comercio electrónico o ventas por Internet, solo el $45 \%$ cuenta con una página Web, pero las orientaciones de estos sitios no tienen claramente definido su objetivo de mercado, la mayoría están orientadas solo a la presentación del portafolio de productos, servicios e información institucional (Chaquea, 2009).

Los proyectos colombianos en Internet no califican en la categoría de comercio electrónico, esto se confirma con la revista Business Technology que en julio de 2000 titula en su portada: "125 compañías colombianas ingresaron a la nueva economía". En las páginas interiores, el informe menciona un total de 113 proyectos: 16 son de multinacionales latinoamericanas, uno es americano y 96 son colombianos. Ninguno de los 96 sitios Web colombianos acepta medios de pago vía Internet.

Actualmente la mayoría de las empresas del sector de refrigeración en Barranquilla están haciendo presencia en la Web, muchas de estas tienen identificado su mercado objetivo, pero ninguna ha incursionado en la modalidad de comercio electrónico. Concretamente nuestro objeto de estudio presenta estas características, es decir, se ubica en la categoría de las Pymes que no realizan comercio electró- 
nico, ni ningún tipo de transacción por la Web, aunque cuentan con un sitio Web, pero este no muestra adecuadamente su portafolio de servicios y no tiene claro la población objetivo.

\section{El Marketing y las herramientas Web}

Desde la aparición masiva de Internet en el mundo empresarial, por los años 90, el marketing comenzó a utilizar este nuevo y revolucionario medio para llegar a los consumidores; el marketing en línea, hace referencia a la estrategia de marketing más tradicional en Internet, utilizando la propia Web como herramienta de marketing, portales, introduciendo en ellos formatos publicitarios. El marketing online es un tipo de estrategia de marketing en constante evolución y que experimenta rápidos cambios en la forma en que se desarrolla y en sus elementos tácticos, entre los cuales se destacan:

Los portales corporativos son sitios web cuyo objetivo es ofrecer a los usuarios un punto de acceso e información a los diversos servicios y/o productos que ofrece o comercializa una determinada empresa. Es como una gran puerta de entrada a la empresa a través de Internet, para los usuarios interesados en obtener información de esta, cumpliendo las siguientes funciones:

- Publicitaria: sirve de soporte para desarrollar acciones publicitarias a través de acciones y anuncios disponibles en distintos formatos.

- Comercial: permite realizar la venta de los productos mediante técnicas de comercio electrónico.
- Producto: el propio portal corporativo es un producto más de la empresa que debe competir con los sitios Web de la competencia para captar clientes que lo visiten.

De igual forma, el marketing online incorpora una serie de estrategias:

- Estrategia basada en relación comercial: ofrece al cliente incentivos económicos, que este no obtiene a través de los canales tradicionales.

- Estrategia basada en el valor agregado: atrae al visitante hacia el portal corporativo a través de introducir en este elemento de contenido que añadan valor desde el punto de vista del usuario.

- Estrategia basada en el marketing viral: convierte al usuario en prescriptor del portal corporativo o sitio Web, intenta conseguir la satisfacción del cliente, con alguno de los elementos que se encuentran en el portal.

- Estrategia de posicionamiento natural en buscadores: los portales corporativos buscan obtener un buen posicionamiento en los resultados de los buscadores, ya que estos rastrean en la Web para generar las bases de datos que contienen información de páginas en Internet.

Actualmente se utilizan los sitios web como soporte para introducir en estos formatos publicitarios, tratando de consolidar la publicidad en diferentes sitios o portales, ya sean propias de la empresa que presta el servicio de marketing o empresas intermediadoras (enlaces). 
El marketing de afiliación, la que la empresa que quiere realizar la acción de marketing busca una página web para poner sus anuncios en ella, de la siguiente manera; los afiliados que ceden su espacio para contener anuncios y así obtener ingresos por ellos; los anunciantes que buscan lugares estratégicos para promocionar sus anuncios y las empresas que se dedican a gestionar la afiliación, ponen a disposición tanto de afiliados como de anunciantes la posibilidad de encontrarse, llevan a cabo toda la gestión, contabilizan visitas, clics, registros y las ventas procedentes de las visitas en tiempo real (Galeano, 2013).

Enlaces Patrocinados. Es un tipo de publicidad que se basa en ciertas palabras claves relacionadas con la actividad del anunciante, y donde el anuncio, contiene un enlace que apunta a la página web del anunciante; su objetivo es aumentar el tráfico hacia la página web de este.

Marketing en buscadores. Los principales buscadores como Google, Yahoo e Internet Exploret, utilizan sistemas de búsquedas patrocinadas, en las cuales las empresas que desean patrocinar la búsqueda ofrecen cantidades de dinero para aparecer en el primer lugar, cuando un usuario realice una búsqueda que incluye una palabra clave; imponiéndose el pago por clic, en el cual las empresas solo pagan cuando alguien hace clic en el enlace.

Marketing en Portales Sociales. Son utilizados con éxito para llevar a cabo actividades de marketing, dando paso a un nuevo tipo de marketing que explota la información de los perfiles de los usuarios y sus relaciones con otros miembros; distinguiéndose dos tipos: marketing en webs sociales de éxito y marketing en mundos virtuales sociales.

\section{Usos Comerciales de la Web}

Internet transformó la educación, la economía e innovó nuestras organizaciones, hasta el punto de hacerla parte esencial e indispensable de los procesos de negocios dentro de la misma; esta herramienta ha tenido un impacto importante en las relaciones de las empresas con entidades como bancos, proveedores y clientes. De acuerdo con Suárez (2011), Internet permite explorar nuevos nichos de mercados, incrementa la accesibilidad, el almacenamiento y distribución de la información; permite disminuir los costos de las transacciones a las que las organizaciones estaban acostumbrados a realizar, haciendo uso de agencias especializadas.

En Internet las barreras de tiempo, distancia y forma se rompen y las empresas son capaces de negociar las ventas de bienes y servicios las 24 horas del día, 7 días a la semana, 365 días al año con consumidores de todo el mundo. De esta manera, la implementación de la Web 2.0, permite la creación e innovación de modelos de negocios como e-commerce, CMR, incluso el uso de redes sociales ha permitido cerrar la brecha que existía entre países, acercándose más al consumidor, comprador y cliente final (Suárez, 2011). 


\section{Herramientas Web}

Para hablar de herramientas Web, debemos ir un poco atrás para conocer cómo era la Web y como es en la actualidad, iniciamos con la Web 1.0 donde las páginas Web eran documentos estáticos y que no se actualizaban, después evolucionamos a la Web 1.5 en esta etapa las páginas son hechas dinámicamente utilizando bases de datos, actualmente nos encontramos en la Web 2.0, en la cual las aplicaciones tradicionales dieron paso a nuevas aplicaciones dinámicas enfocadas al usuario final, convirtiéndose en grandes contribuidores y gestores del contenido de estas, contamos con sitios Web colaborativos que han remplazado las software de escritorio.

Redes sociales: Las Redes son formas de interacción social, definida como un intercambio dinámico entre personas, grupos e instituciones en contextos de complejidad. Un sistema abierto y en construcción permanente que involucra a conjuntos que se identifican en las mismas necesidades y problemáticas y que se organizan para potenciar sus recursos (Martos, 2010).

\section{Comercio Electrónico (E-Commerce)}

Está cambiando la forma de competir, la velocidad de acción y la modernización de las interacciones, los productos y los pagos de los clientes a las empresas y de estas a los proveedores (Rodríguez, Rivera \& Nakazono, 2013).

El comercio electrónico consiste en la compra y venta de productos o de servicios a través de medios electrónicos, como Internet. La cantidad de comercio llevada a cabo electrónicamente ha crecido extraordinariamente debido a la propagación del Internet. Una gran variedad de comercio se realiza de esta manera, estimulando la creación y utilización de innovaciones como la transferencia de fondos electrónica, la administración de cadenas de suministro, el marketing en Internet, el procesamiento de transacciones en línea (OLTP), el intercambio electrónico de datos (EDI), los sistemas de administración del inventario, y los sistemas automatizados de recolección de datos.

El comercio electrónico es más que solo comprar y vender productos en línea. En vez de eso, abarca todo el proceso en línea de desarrollo, mercadotecnia, venta, entrega, servicio y pago de productos y servicios negociados en pagos globales interconectados de clientes, con el apoyo de una red mundial de socios de negocios (O'Brien \& Marakas, 2007).

El comercio electrónico utiliza tres elementos principales; y sus tipos se establecen en función de sus actores participantes; se distinguen tres tipos básicos de intercambios comerciales.

Comercio entre empresas o B2B. Se refiere al espectro completo del comercio electrónico que ocurre entre dos organizaciones. Además de otras actividades, el comercio B2B incluye compras y procuración, administración de proveedores, inventarios, canales de distribución, 
actividades de ventas, pagos, así como servicio y soporte (Rayport \& Jaworski 2006).

En ese sentido Lara-Navarra y Martínez-Usero (2002), afirman que esta categoría incluye mercados de negocios electrónicos y vínculos directos de mercado entre empresas; ha sido utilizada por muchos años, particularmente haciendo uso de EDI sobre redes privadas o redes de valor agregado; entre sus pioneros citamos a Chemdex, fastparts y freemarkets.

El B2B ha venido impulsado fundamentalmente por la creación de portales para agrupar compradores. Así, encontramos, por ejemplo, portales de empresas de alimentación, químicas u hostelería, entre otros. Las compañías se agrupan para crear dichas páginas aglutinando fuerzas lo que les permite negociar en mejores condiciones. Entre las ventajas que aporta el B2B para los compradores se pueden resaltar: la posibilidad de recibir mayor número de ofertas y la no personalización de la compra con lo que se evitan posibles tratos de favor.

Comercio entre empresa y consumidor o B2C. Es el comercio electrónico que realizan las empresas con los consumidores. (Amazon.com, y Yahoo.com) Se puede comparar con la venta al detalle de manera electrónica. Esta categoría ha tenido gran aceptación y se ha ampliado sobre manera gracias al World Wide Web, ya que existen diversos centros comerciales (Marketplace) por todo Internet.

O’Brien y Marakas (2007), afirman que, en esta forma de comercio electrónico, las empresas deben desarrollar mercados electrónicos atractivos para vender productos y servicios a los consumidores. El éxito de B2C pasa por el aseguramiento de los sistemas de pago a través de tarjeta de crédito, si bien en muchos casos se da la posibilidad de otras formas de pago como contra reembolso, en efectivo o la utilización de servicios proporcionados por otras empresas como PayPal.

Comercio entre Consumidor a Consumidor o C2C. Rayport y Jaworski (2006), dicen que comprenden transacciones entre dos o más consumidores, estos intercambios incluyen o no la participación de terceros, como en el caso de las subastas o intercambios. Además realizan otras actividades como: anuncios clasificados, juegos, empleos, comunicación con base en la Web y servicios personales.

Comercio entre Consumidor a Negocio o C2B. Los consumidores pueden unirse para formar grupos de compradores y presentarse como tal a los negocios; según Rayport y Jaworski (2006), por lo general estos grupos son incentivados por motivaciones económicas, como agregadores de demanda.

\section{Ventajas del E-commerce para las empresas}

Reduce los canales de comercialización, permitiendo que la distribución sea eficiente al reducir sobrecostos derivado de la uniformidad, automatización e integración a gran escala de sus procesos de administración; de ahí que puede disminuir el tiempo que se tardan 
en realizar las transacciones comerciales, incrementando la eficiencia de las empresas (Sellers \& Azorín, 2011).

Actualmente, la mayoría de las empresas utiliza la Web para mostrar información tanto de la compañía como de sus productos y/o servicios, mediante comunicaciones internas, así como con otras empresas y clientes; sin embargo, la naturaleza interactiva de la web ofrece otro tipo de beneficios conducentes a desarrollar las relaciones con los clientes; este potencial para la interacción facilita las relaciones comerciales, fortaleciendo el soporte al cliente, hasta un punto que nunca hubiera sido posible con los medios tradicionales.

Fidelización de sus clientes mediante un diálogo asincrónico que sucede a la conveniencia de ambas partes; esta capacidad ofrece oportunidades sin precedentes para ajustar con precisión las comunicaciones a los clientes individuales (Zorrilla, 2009), facilitando que estos soliciten tanta información como deseen; de esta manera obtener información relevante de los clientes, con el propósito de servirles de manera eficaz en las futuras relaciones comerciales. En ese sentido la Web permite competir sobre la base de la especialidad; Garcés y Rojas (2014) manifiestan que, en lugar de hacerlo mediante el precio, ya que, desde el punto de vista del mercadeo, rara vez es deseable competir tan solo en función del precio.

El comercio electrónico intenta satisfacer las necesidades de los clientes con base en lo que buscan, lo que quiere decir, que el precio depende de la valorización del cliente, y no de los costos; tales oportunidades surgen cuando lo ofrecido se diferencia por elementos de mercadeo distintos al precio, lo cual genera beneficios cargados de valor (Zorrilla, 2009). El uso empresarial de la Web reduce errores, tiempo y sobrecostos en el tratamiento de la información.

De igual modo, Clarenc (2011), afirma que los proveedores disminuyen sus costos al acceder de manera interactiva a las bases de datos de oportunidades de ofertas, enviar estas por el mismo medio, y, por último, revisar de igual forma las concesiones. En concordancia facilitan la creación de mercados y nuevos segmentos, así como el incremento en la generación de ventajas en las ventas, la mayor facilidad para acceder a nuevos mercados, quitando los límites geográficos y así llegar a ellos con mayor rapidez

\section{Usos del Comercio Electrónico}

Este puede utilizarse en cualquier entorno en el que se intercambien documentos entre empresas: compras o adquisiciones, finanzas, industria, transporte, salud, legislación y recolección de ingresos o impuestos. Ya existen compañías que utilizan el comercio electrónico, de acuerdo con Clarenc (2011) desarrollaran los aspectos siguientes:

- Creación de canales nuevos de marketing y ventas.

- Acceso interactivo a catálogos de productos, listas de precios y folletos publicitarios. 
- Venta directa e interactiva de productos a los clientes.

- Soporte técnico sin interrupciones, permitiendo que los clientes encuentren por sí mismos, y fácilmente, respuestas a sus problemas mediante la obtención de los archivos y programas necesarios para resolverlos.

A través del comercio electrónico se logra el intercambio de actividades empresariales entre socios comerciales; obteniendo los siguientes beneficios: reducción del trabajo administrativo, transacciones comerciales más rápidas y precisas, acceso más fácil y rápido a la información, y reducción de la necesidad de reescribir la información en los sistemas de información. Dentro de los tipos de actividad empresarial que podrían beneficiarse mayormente de la incorporación del comercio electrónico encontramos:

- Sistemas de reservas. Centenares de agencias dispersas utilizan una base de datos compartida para acordar transacciones.

- Stocks. Aceleración a nivel mundial de los contactos entre proveedores de stock.

- Elaboración de pedidos. Posibilidad de referencia a distancia o verificación por parte de una entidad neutral.

- Seguros. Facilita la captura de datos.

- Empresas proveedoras de materia prima a fabricantes. Ahorro de grandes cantidades de tiempo al comunicar y presentar inmediatamente la información que intercambian.
La toma de decisiones en el Comercio Electrónico

Las empresas están reconociendo que el éxito será para aquellos que puedan ejecutar estrategias virtuales y no virtuales, que unan el mundo físico con el virtual. Diferentes empresas necesitarán seguir caminos muy diversos al decidir con cuanta rapidez o libertad integrarán sus iniciativas de Internet con sus operaciones tradicionales (Lirios, 2012).

Al incursionar en los modelos de negocio de comercio electrónico se debe tener en cuenta a que grupos de clientes se debe servir, analizar los competidores y asociados de negocios potenciales. Se debe decidir la propuesta de valor o grupo de valores que se va a ofrecer al segmento, la oferta del producto específico, el sistema de recurso para entregar los beneficios, el modelo financiero a seguir. Es importante tener identidad propia y establecer como el cliente va a percibir el negocio; diseñar la estrategia para llegar al mercado, es decir, establecer el proceso de implantación, este proceso enmarca el sistema de entrega y la innovación para el servicio de los clientes.

\section{Metodología}

Esta propuesta está enmarcada en la línea de investigación innovación empresarial y fundamentada en una investigación propositiva y aplicada, ya que se busca que con este estudio las empresas puedan aprovechar al máximo los elementos que nos ofrece la Web y todos los medios tecnológicos que tenemos a nuestro alcance para la toma de decisiones estra- 
tégicas, y de esta forma lograr un mayor posicionamiento en el mercado y así mantenernos competitivos para subsistir.

Dentro de la investigación se utilizarán los siguientes métodos: Analítico, en esta fase se pretende conocer muy detalladamente al objeto de estudio, particularmente a las empresas escogidas estudiando cada una de las partes integrantes del mercadeo y el comercio electrónico. Cualitativo basándonos en la observación y análisis de hechos enmarcados en variables cualitativas e información primaria.

\section{Modelo de negocio propuesto}

A partir del comercio electrónico como eje central, se fortalecerá la relación con los clientes, manteniéndolos actualizados en tiempo real del portafolio de servicios que ofrecen las empresas objeto de estudio, a través del catálogo digital, impulsando el marketing online con lo que se posicionará la marca inicialmente a nivel nacional.

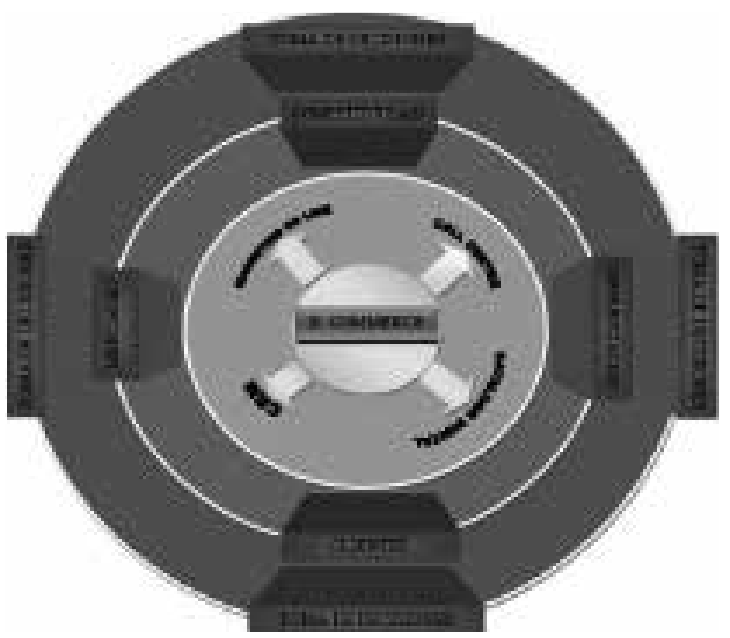

Figura 1. Modelo de E-commerce Fuente: Elaboración propia
En la figura anterior, se presenta el modelo obtenido como resultado del análisis de cada una de las teorías estudiadas, y de las tecnologías a aplicar.

La articulación de estas herramientas se convertirá en un factor diferenciador, lo que mejorará el mercadeo y con ello a su vez la competitividad hasta alcanzar la fidelidad de los clientes y la conquista de nuevos mercados mediante la mejora continua evidenciada por el fortalecimiento de la toma de decisiones. En ese sentido el marketing digital cuenta con instrumentos para establecer una relación continua entre la empresa y sus clientes, reuniendo las claves del marketing directo. A continuación, describimos los componentes del comercio electrónico que se proponen en el modelo

- El marketing digital es en sí mismo un sistema de marketing: no se trata solamente de un medio publicitario, ni una técnica de venta, sino que además es una vía de marketing apta para encontrar mercados, estimular la demanda y ofrecer productos a los consumidores, de esta manera se involucran todas las herramientas de marketing, para conquistar al cliente potencial.

- Permite establecer un sistema de comunicación con el mercado: se hace con el fin de estimular e informar las respuestas de compra de los consumidores por medio de instrumentos de comunicación en lugar de personal y puntos de venta.

- Ofrece una retroalimentación medible y estable: Las reacciones de la audiencia a la que se dirigen las acciones de marketing 
son medibles, ya sea a través del nivel de audiencia del sitio Web, de los mensajes de respuesta o su decisión de compra (Equipo de tutores, 2010, p.4); de esta manera se puede saber en qué momento la empresa debe retirar el producto del mercado.

El CRM como importante tecnología de marketing en Internet, es un proceso interactivo, en el cual la información sobre el cliente se transforma en provechosas relaciones con este. Mediante el uso de esta técnica, se acelera la toma de decisiones administrativas, aumentado su utilidad, fortalece al personal que está en contacto con el cliente a los departamentos de marketing y ventas (Guzmán \& Montana, 2014).

Administrar todo el rango de las relaciones con los clientes implica dos objetivos: uno, proporcionar a la organización y a todos sus empleados de contacto con el cliente una visión única y completa de cada cliente en cada punto de contacto y en todos los canales; y, dos, proporcionar al cliente una visión completa y única de la empresa y sus canales ampliados (O’Brien \& Marakas, 2007).

Catalogo Digital. Una empresa que desee vender productos en Web, debe tener una lista, o un catálogo en línea de sus productos, este módulo incluye una herramienta de base de datos que permite construir un catálogo en línea personalizado; la complejidad y sofisticación del catálogo varía dependiendo del tamaño de la empresa y sus líneas de productos (Laudon \& Guercio, 2009).
Los catálogos digitales nos permiten mostrar el portafolio de servicios o productos que ofrece la empresa, de esta manera mantiene actualizados los productos ofrecidos por una empresa, tienen la ventaja de que se pueden modificar constantemente y disminuyen los costos de impresión y envío de estos a los clientes que se encuentran en otras ciudades.

Call Center. Esta técnica simple de utilizar nos ayuda a aumentar las ventas y permite generar reportes en línea de todas las llamadas recibidas, de esta forma, si algún cliente no pudo realizar la llamada, la empresa la puede devolver en cualquier momento, para ello se debe habilitar una opción que le permite al visitante llamar a la empresa sin que le cueste nada.

\section{Conclusiones}

Es importante que las empresas establezcan una relación compenetrada con sus clientes, buscando siempre la plena satisfacción de estos, para incentivar en ellos la fidelidad hacia la empresa, un cliente feliz es un cliente que puede promocionar a la compañía.

Internet es una plataforma tecnológica, que ha tenido mucha fuerza impactando no solo la comunicación, sino revolucionando la manera de hacer negocios, gracias a sus características y gran aceptación, en el cual su eje fundamental es el comercio electrónico, ya que esta mejora significativamente la competitividad de la empresa.

Implementar comercio electrónico no debe ser visto como un reto, sino como una oportu- 
nidad de negocio para optimizar los procesos, mejorar la comunicación con los proveedores, clientes, mediante las transacciones que fluyan entre las partes.

Los clientes deben verse como el activo más importante de la empresa, por ello se debe mantener los clientes a través de programas de fidelización, porque es más rentable mantener un cliente que buscar nuevos potenciales.

\section{Referencias}

Berenguer, J. \& Ramos, J. (2003). Negocios Digitales: competir usando tecnologías de información. Ediciones Universidad de Navarra.

Chaquea, L. (2008). Uso del Internet y nuevas tecnologías como herramientas para las PYMES colombianas exportadoras. Recuperado en http://www.unionlideres. com

Clarenc, C. (2011). Nociones de Cibercultura y Periodismo. $1^{\text {a }}$ ed.

Equipo de tutores (2010). Marketing digital. Editorial eLearning.

Galeano Sánchez, J. C. (2013). El fenómeno del consumo, entendido desde su historicidad y su impacto social. Ad-Gnosis, 2(2), 129-146.

Garcés, B. \& Rojas, M. (2014). Investigación de mercado para la creación de una tienda virtual de juguetes Brenda Carolina (Trabajo de grado). Universidad San Buenaventura.
Guzman, A. \& Montana, K. (2014). Desarrollo de una Estrategia CRM para la Implementación de un Plan de Fidelización de Clientes en Sofalca Comercializadora (Trabajo de grado). Universidad Nueva Granada.

Lara-Navarra, P. \& Martínez-Usero, J. (2002). Del comercio electrónico a la administración electrónica: tecnologías y metodologías para la gestión de información. El profesional de la información, 11(6), 421-435.

Laudon, K. \& Guercio, C. (2009). E-commerce: negocios, tecnología, sociedad. $4^{\text {a }}$ ed. Editorial Prentice-Hall.

Lirios, C. G. (2012). Estructura híbrida de los determinantes sociodemográficos del consumo electrónico. Revista de Psicología Gepu, 3(2), 43-53.

Martos, E. (2010). Análisis sobre las nuevas formas de comunicación a través de las comunidades virtuales o redes sociales. (Trabajo de grado). Universidad Politécnica de Valencia.

O’Brien, J. \& Marakas, G. (2007). Sistemas de información Gerencial. $7^{\mathrm{a}}$ ed. McGraw-Hill Interamericana.

Rayport, J. \& Jaworski, B. (2006). E-commerce. $1^{\text {a }}$ ed. Editorial McGraw-Hill Interamericana.

Rodríguez, E. C., Rivera, M. L. G. \& Nakazono, R. L. (2013). El comercio electrónico: en la era de la globalización. Contribuciones a la Economía, 7. 
Sellers, R. \& Azorín, A. (2011). El Comercio Electrónico y el Futuro del Canal de Distribución Turístico. Investigaciones Europeas de Dirección y Economía de la Empresa, 7(1), 13-36.

Suárez, D. (2011). Influencia de los sistemas de información en las organizaciones. Revista Pensamiento Americano, 2(6), 31-34.
Zorrilla, P. (2009). El equilibrio natural de la economía de mercado y hacia el e-conomy. El analista económico-financiero. Recuperado de https://elanalistaeconomicofinanciero.blogspot. com.co/2009/03/el-equilibrio-natural-de-la-economia-de.html

125 Compañías Colombianas ingresaron a la nueva economía (2000). Revista Business Technology. Edición aniversario. 